\title{
Cryo-EM and protein-protein docking reveal myosin loop 4 contacts actin and tropomyosin on thin filaments
}

\author{
M Doran ${ }^{1}$, W Lehman ${ }^{1}$, E Bullitt ${ }^{1}$, M Rynkiewicz $^{1}$, E Pavadai $^{1}$, M Regnier $^{2}$, M Geeves $^{3}, \mathrm{~J} \mathrm{Moore}^{4}, \mathrm{~J}$ \\ Walklate ${ }^{4}$ \\ ${ }^{1}$ Boston University, Boston, MA, ${ }^{2}$ University of Washington, Seattle, WA, ${ }^{3}$ University of Kent, \\ United Kingdom, ${ }^{4}$ University of Massachusetts Lowell \\ mttdoran@bu.edu
}

Cardiac muscle contraction occurs when thin filaments slide past thick filaments driven by the action of the molecular motor, myosin. Projecting from thick filaments, myosin heads bind to thin filament actin and undergo ATPase-associated conformational changes to propel the filaments to move past one another. Tropomyosin, a coiled-coil protein that wraps around actin as a continuous cable, regulates this process by sterically controlling myosin's ability to bind to actin. Although several actomyosin structures have been proposed, the structure describing cardiac actin - myosin interactions including tropomyosin has not been solved. Here, we present the structure of the cardiac actomyosin-tropomyosin complex in the nucleotide-free state, solved to a resolution of 4.2 $\AA$ using cryo-electron microscopy and helical reconstruction. The atomic model derived from this reconstruction shows a strong, mostly hydrophobic interface between actin and myosin that is consistent with previously determined non-cardiac structures. However, we were able to obtain additional residue-residue discrimination, by combining our cryo-EM structure with results of protein-protein docking methods, revealing novel electrostatic interactions between myosin loop 4 (363-376) and tropomyosin. This hybrid method shows that the tip of myosin loop 4, most notably residue Arg369, forms distinct interactions between successive tropomyosin segments on actin protomers. We propose that these favorable contacts compete with and displace ones normally found between actin and tropomyosin in the absence of myosin, playing a vital role in the transition from the relaxed to contracted states. The impact of these structural studies is twofold: (1) they suggest that the favorable myosin loop $4-$ tropomyosin interaction restructures the thin filament during the actin-myosin ATPase cycle; (2) they lay a foundation for understanding molecular mechanisms by which disease-rendering point mutations perturb normal actin-myosin-tropomyosin binding.

Acta Cryst. (2020). A76, a99 